\title{
Fiber and Wavelength Open Access in WDM- and TWDM Passive Optical Networks
}

\author{
Abhishek Dixit, Marlies Van der Wee, Bart Lannoo, Didier Colle, Sofie Verbrugge, Mario Pickavet and \\ Piet Demeester, Ghent University-iMinds
}

\begin{abstract}
There has been a large global effort to innovate and design optical access technologies that can accommodate the requirements emerging from a colossal increase in data rates. Currently time and wavelength division multiplexed passive optical networks (TWDM-PONs) and WDM-PONs have been foreseen as the main candidates for next-generation access systems. Due to current business modeling trends and possible regulatory obligations, these networks should also support open access, which refers to the sharing of a network infrastructure among different network entities in a non-discriminatory way. By sharing the (bottleneck) infrastructure facility, open access reduces the entry barrier for a network entity. This opens doors for a multi-provider scenario, which leads to competition among network players and can significantly reduce the price of services. Opening up the network, however, entails new architectures. In this paper, we propose novel architectures to support open access at fiber and wavelength level for WDM- and TWDM-PON. These architectures, however, differ significantly in terms of their cost (capital and operational expenditures). We compare the proposed architectures with regard to their cost and analyze the impact of adoption levels (percentage of users subscribed) and customer churn rate (how often the customers change network) on the cost of the architectures.
\end{abstract}

\section{INTRODUCTION}

Open access [1] is a well-established theme that allows competition and as such supports new business models to make fiber- to- the-home (FTTH) networks an economically viable solution. FTTH networks can deliver high bandwidth to customers, and thus are future-proof solutions. They, however, require a high initial investment to deploy fiber in the field, and it is not possible to recoup the investments made within the usual depreciation periods of e.g. 5 to 10 years. Hence, a natural solution is to share network infrastructure (fiber and equipment) among multiple network entities. Sharing network infrastructure ensures that not every network entity has to make huge capital expenditures (CapEx) before being able to serve users. This reduces the barrier for network entry, encourages competition, and consequently, reduces the price of services.

For its success, open access entails the sharing to be non-discriminatory, requires new business models and revenue-flow paths, and necessitates novel architectures to stimulate a multitude of services at the user's end in a seamless way. In this paper, we focus on architectural challenges to open a network.

Open access can be offered at different layers (section III) depending on how a user selects a specific network entity, e.g., by selection of a fiber, wavelength, or a packet field (Ethernet address, VLAN tag, MPLS, IP). This classifies open access as fiber, wavelength, and bit-stream open access. While the first two flavors of open access require new architectures, the latter can simply be implemented by providing a slice of network resources to a network entity. This slicing can be implemented at layer 2 (VLAN), layer 2.5 (MPLS) or layer 3 (IP) by emerging cutting-edge technologies like software defined networking and network virtualization. Hence, the bit-stream open access can be implemented without adapting architectures, and consequently, is less challenging. This paper focuses on only fiber and wavelength open access, which require new architectures, but provide network entities a higher degree of flexibility in designing their access network compared to bit-stream open access.

In this paper, we propose novel architectures (section IV) for fiber and wavelength open access in next generation access systems. As specific examples, we choose time and wavelength division multiplexed passive optical network (TWDM-PON) [2] and WDM-PON [3], which have been chosen by the full service access network (FSAN) group as the candidates for next generation access systems, or next generation-PON2 in FSAN terminology. We also evaluate the CapEx and operational expenditures (OpEx) of these architectures in section V. In addition, we factor the variability in the cost evaluation due to different adoption levels (percentage of the users subscribed) and customer churn rate (how often the users change network). 


\section{NEXt GENERATION-PASSIVE OPTICAL NETWORKS2}

WDM- and TWDM-PON scale sustained bandwidth per residential customers and will potentially serve as the candidates for NG-PON2. WDM-PON increases the capacity of the conventional PONs (mainly time division multiplexed (TDM), e.g., EPON, GPON, XGPON) by using a wavelength layer in conjunction with a passive optical distribution network (ODN). Out of many flavors of WDM-PON, we assume wavelength routed WDM-PON, which uses a cyclic arrayed waveguide grating (AWG) in the remote node (RN, at the cabinet) to multiplex/demultiplex wavelengths and route a wavelength pair (up- and downstream) to each optical network unit (ONU, i.e., the equipment at the user's premises), see Figure 1. Cyclic AWGs allow access of different up- and downstream wavelength bands. The ONU uses a broadband receiver (to be able to receive any wavelength used by the WDM-PON) and a tunable transmitter to minimize an inventory of ONUs at different wavelengths.

TWDM-PON combines the flexibility of TDM in resource allocation with an added capacity of WDM. TWDM-PON uses a power splitter (PS) at the RN, which broadcasts wavelengths to all ONUs (Figure 1). The ONU now requires a tunable receiver and a security layer as multiple wavelengths are available at its input. Furthermore, it uses tunable transmitters like in WDM-PON.

\section{OPEN ACCESS FLAVORS}

In open access, multiple network entities serve at different functional levels and thus do not bear the financial baggage of end-to-end network provisioning, especially network infrastructure investment. Network provisioning can be conceptually separated into three roles, typically taken up by different entities:

- Physical infrastructure provider (PIP) responsible for installation of the physical infrastructure (implying trenches, conduits, ducts, fiber, housing).

- Network provider (NP) - responsible for all active equipment between the users and the central office (CO), e.g., optical line terminals (OLTs, i.e., CO equipment) and ONUs.

- Service provider (SP) - supply of services (telephony, IPTV, broadband Internet, mobile backhauling) and installation of service specific equipment (e.g., set-top box for Digital TV).

- This separation is based on the technical and economic nature of the roles [4]. For example, providing physical infrastructure requires high CapEx, low OpEx, and low economies of scale. Network or service provisioning entails high OpEx and high economies of scale. Note that we have not assigned the role of providing passive equipment (such as PSs and AWGs) to any functional entity, as it depends on the specific open access scenario (see later).

These different functional entities - PIP, NP and SP - participate and coexist in an open access scenario. This warrants defining the interfacing between these functional players to assure compatible service delivery. Here, we can clearly identify two open access interfaces (OAI): PIP-NP and NP-SP. In the first interface, multiple NPs exist over a common PIP, and in the second, multiple SPs exist over a common NP. The latter interface can be opened by sharing logical space (OSI layer 2 and above) among different SPs by using an element on the OSI network layer 2 (Ethernet) or layer 2.5/3 (MPLS, IP), also referred to as bit-stream open access.

Opening the PIP-NP interface is more complex as it involves adaptations in the architectures and introduces new components. This interface can be opened by fiber and wavelength open access.

Fiber open access - Opening at the fiber layer means that a user selects an NP through a fiber. This provides access to different NPs at the RN, stimulating multiple NPs in the same geographical area, e.g., in the FTTH network of Amsterdam, where KPN and BBNed are both NP within the same geographical area, and in France, where a law [4] obliges the PIP to deploy multiple fibers to every building.

Wavelength open access - Opening at the wavelength layer means that a user can select an NP using one or more dedicated wavelengths. Wavelength open access can be used to give access to different NPs at RN or at OLT, also facilitating a multi-NP scenario. Currently, wavelength open access is actively considered in the Open Lambda Initiative [6]. 


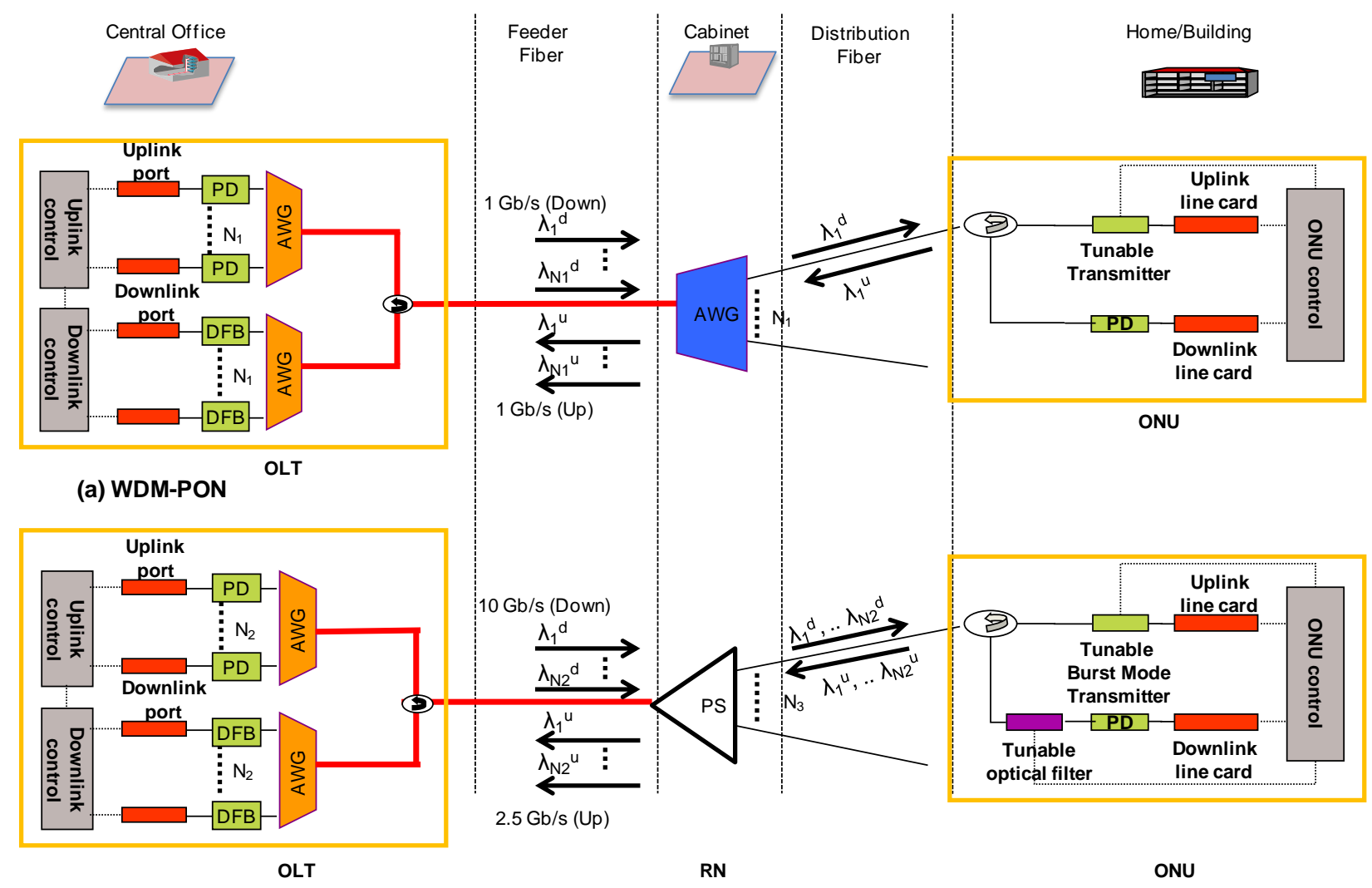

(b) TWDM-PON

Figure 1: WDM- and TWDM-PON architectures. Abbreviations used in the figure: PD: photodiode, DFB: distributed feedback laser. In this paper, $N_{1}=32, N_{2}=4, N_{3}=512$.

\section{OPEN ACCESS ARCHITECTURES FOR NG-PON2}

We discuss architectures for fiber and wavelength open access in WDM- and TWDM-PON, according to the interface where network access is opened. The interface allowing open access makes a fundamental difference to the ownership of the network, and the characteristics of the NPs.

\section{A. RN Interface}

We present architectures to open the network at the $\mathrm{RN}$ interface in Figure 2. There is no explicit difference between WDM- and TWDM-PON for opening at the RN interface. The network can be opened at the fiber level (Figure 2 (a) and (b)) or wavelength level (Figure 2 (c)). To allow open access, the architectures may require an additional interface point, referred to as a point of unbundling (PoU). The PoU can be defined as the first point at which different (at least 2) NPs or SPs are brought together on the same device (e.g. fiber cable, splitter, AWG, Ethernet switch).

Fiber open access: Figure 2 (a) depicts a scheme in which each ONU has one distribution fiber (DF), which is shared (unbundled) among multiple NPs using the optical distribution frame (ODF) at the RN. Thus, every time a user wants to change its NP, fiber re-patching is required at the ODF, increasing OpEx. This scheme is preferred in the fiber-lean scenario, as it requires only a single DF per ONU.

In Figure 2 (b), each ONU has a dedicated DF to reach every NP and the selection of an NP is done through the fiber switch at the ONU. To support this, a fiber-rich deployment is needed. The cost for installing a couple of extra fibers is negligible [7] in comparison to the trenching and ducting costs, and should therefore be considered anyhow when setting up a deployment planning.

In fiber open access, the PIP deploys only fiber and remains technology agnostic. This ensures freedom to NPs to choose its technology, leading to heterogeneous NPs. Moreover, as the NPs have a separate fiber infrastructure, they have complete isolation from other NPs. However, the disadvantages are that sharing is limited to only fiber infrastructure and the migration of a user to a different NP is restricted as it entails changing users' equipment to adapt to a different technology.

Wavelength open access: Figure 2 (c) presents a wavelength open access scheme, which uses a 


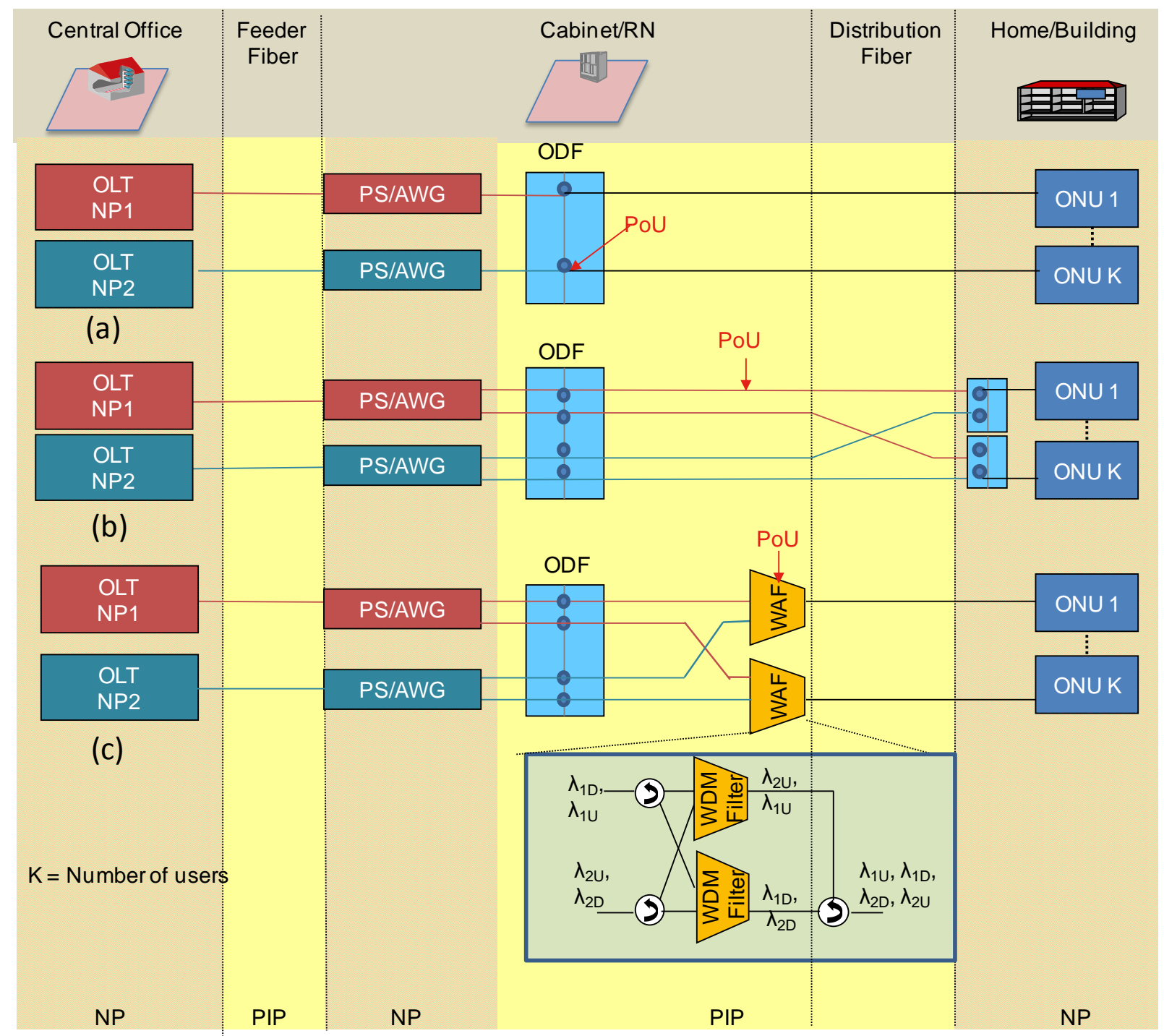

Figure 2: Open access schemes at the RN interface: a) Fiber open access $(P o U=O D F)$ b) Fiber open access $(P o U=D F)$ c) Wavelength open access $(\mathrm{PoU}=$ WAF $)$. Different network players, PIP and NP, own different parts of the network, which is depicted using colored patterned segments.

wavelength access filter (WAF) to provide access to different networks based on wavelengths. Different windows of wavelengths (shown in the figure as $\lambda_{1 \mathrm{U}}$, $\left.\lambda_{1 \mathrm{D}}, \lambda_{2 \mathrm{U}}, \lambda_{2 \mathrm{D}}\right)$ are used by PON technologies for coexistence, here $U$ and $D$ stand for up- and downstream wavelength and 1 and 2 represent two networks. For example, GPON uses 1290-1330 nm $\left(\lambda_{1 \mathrm{U}}, \mathrm{O}\right.$ band) for upstream and $1480-1500 \mathrm{~nm}\left(\lambda_{1 \mathrm{D}}, \mathrm{S}\right.$ band) for downstream transmission, whereas TWDM-PON will use a different band [2]. Thus, different PON technologies can be differentiated using a WAF, which is composed of WDM filters for up- and downstream direction. Wavelength open access works if the NPs use either different coexisting technologies or different wavelengths within the standard band. This is a promising option for a fiber-lean deployment with no re- patching required in the ODF.

As in fiber open access, the PIP remains technology agnostic and NPs can use heterogeneous technologies.

\section{B. OLT Interface}

The OLT interface can be opened on the wavelength layer to allow NP-PIP OAI (Figure 3 and Figure 4). The main differentiator with the options in Figure 2 is that now the PIP should own the entire passive infrastructure (physical infrastructure and passive equipment). This is because if one of the NPs owns passive equipment, it can leverage special benefits in its competition against other NPs due to 


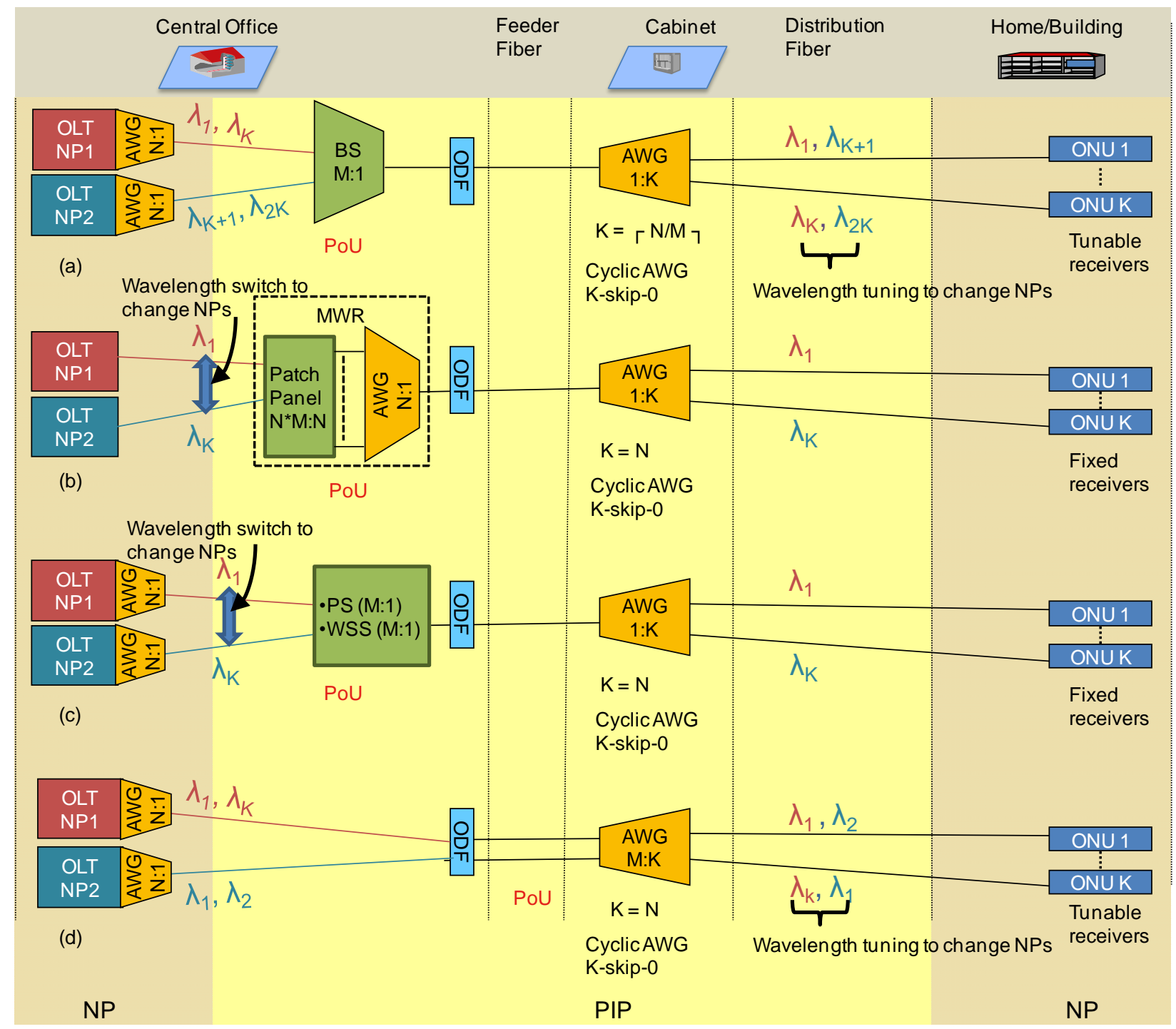

Figure 3: Wavelength Open access schemes at the OLT interface for WDM-PON with point of unbundling (PoU) as: a) BS b) MWR c) PS/WSS d) FF. Wavelength mapping shows how OLT connects with ONU1 and ONUK and N = number of wavelengths; M= number of NPs; $K$ = number of users.

its ownership of the passive infrastructure. Hence, for the access to be non-discriminatory ${ }^{1}$, an actor should not be allowed to have an ownership of the facility that it is using to compete against other players. Moreover, as the PIP owns passive infrastructure, it does not remain technology agnostic; this confines all NPs to use a homogeneous technology, curbing their degree of freedom. The major advantage however is that it allows easier customer migration. Since all NPs use the same technology, a user does not need to

\footnotetext{
1 The non-neutral environment in which a single actor participates in both a particular layer and the layer on top of that, but still allowing the co-existence of other actors on top of its own passive infrastructure/network is generally referred to as unbundling.
}

change its ONU and can switch NPs easily.

To make the migration of users even easier, it is assumed that the wavelengths from every NP should reach every user. These architectures are impacted by the technology in consideration, and hence, they differ for WDM- and TWDM-PON.

WDM-PONs - We consider five likely options to implement a PoU in WDM-PONs: band splitter (BS), manual wavelength router (MWR), PS, wavelength selective switch (WSS), and feeder fiber (FF). Figure 3 (a) presents the BS based wavelength open access solution for WDM-PON. The BS combines and distributes the spectrum for different NPs. Since the BS is a static splitter, the NPs are assigned a static 
chunk of spectrum that cannot be rearranged with a varying number of users per NP. To satisfy the condition that the wavelength from every NP can reach every user, a cyclic AWG is assumed at the RN with a free spectral range (FSR) equivalent to a wavelength band per NP. Cyclicity combined with limited FSR allows multiple wavelengths at the output port of an AWG, where each wavelength belongs to an NP. The FSR, and consequently fan out, should be limited to accommodate $\mathrm{K}$ wavelengths ( $\mathrm{K}$ in the best case is $\mathrm{N} / \mathrm{M}$, where $\mathrm{N}$ is the number of wavelengths, and $M$ is the number of NPs). Thus the number of users is now reduced by a factor M (the number of NPs), increasing the cost per user. Tunable receivers are assumed at the ONU to select the NP by tuning the receiver to the right wavelength. Note that in normal (no open access) WDM-PON, the transmitters are already tunable. The receiver, however, is a fixed broadband receiver.

Figure 3 (b) presents the solution in which the PoU is an MWR. The MWR consists of a patch panel and a demultiplexer. Also, note that in this scheme, transceivers from the OLT are connected directly to a patch panel, instead of combined first by a multiplexer. This is to avoid additional insertion losses in multiplexing and demultiplexing. Figure 3 (c) presents the solution in which the PoU can be a PS or WSS. Since an MWR, PS, or WSS can be flexibly configured, these solutions can dynamically allocate the spectrum among NPs. They also remove the need of tunable receivers at the ONUs. In these schemes, the selection of the NP is done by using the right wavelength at the NP. For example, if an ONU wants to move from $\mathrm{NP}_{1}$ to $\mathrm{NP}_{2}$, the NPs should appropriately rearrange their wavelengths usage. However, these solutions also have drawbacks. An MWR based solution requires fiber patching every time a user wants to switch and thus adds OpEx. The scheme with PS as PoU requires all NPs to comply with the maximum output power, wavelength grid, etc; otherwise, an NP can disrupt services of other NPs and violates inter-NP isolation. It additionally requires a test equipment (not shown in the figure) and continuous monitoring of the data stream from different NPs to ensure that all NPs comply with the requirements. The downside of WSSs based PoU is its active, expensive and failure prone characteristics.

To solve these problems, we propose a FF based open access solution in Figure 3 (d). It uses multiple FFs and an M: K AWG at the RN, requiring a fiberrich scenario. Now all NPs can use the entire spectrum. The latent routing property of AWGs, i.e. the two same input wavelengths can never appear out from the same port, prevents any conflict concerning the spectrum use among NPs. The configuration allows every user to receive wavelengths from all NPs, and to tune to the right wavelength. However, the ONUs need to have tunable receivers.

TWDM-PONs - The TWDM-PONs can use the same PoU as WDM-PONs (Figure 4). However, for using FF based PoU, an additional AWG has to be used at the RN, as a PS collides the data on the same wavelength. In TWDM-PONs, the NP selection is always made by tuning the $\mathrm{ONU}$ to the right wavelength. TWDM-PONs experience another constraint in providing open access. The use of a PS at the RN raises security concerns among different NPs. A defective NP can now affect the services of other NPs and a user from a different NP can affect the services of users of other NPs, which makes interNP isolation not per se available in TWDM-PON. Figure 4 (c) therefore presents the secure open access implementation to provide inter-NP isolation. For the illustration of this scheme, we use the FF based scenario as discussed before. However, the technique of providing network isolation can be used in conjunction with all PoU. We use an interleave filter that creates separate NP space in combination of a PS. The users can access different NPs using a patch panel at the location of a building basement. This approach safeguards against a rogue (defective by accident) user and provides higher security against malicious (defective by purpose) users. A malicious user can still theoretically affect the services of other NPs, but can be easily monitored by a CCTV camera at the location of the patch panel and can be suspended by an NP. Moreover, using a patch panel will not incur in OpEx if a user is allowed to slot in its fiber. If, however, the users cannot be expected to do fiber patching, this scheme will increase OpEx.

\section{Cost Evaluation}

In this section, we evaluate CapEx and OpEx of the proposed architectures for open access in WDMand TWDM-PON. For CapEx, we only include costs of the components. The cost of other physical infrastructure, with respect to digging, ducts, and housing, is though quite significant, generally accounting for about $67 \%$ [7] of the overall total cost of ownership; however, it is almost similar [7] and as such negligible when comparing architectures. Regarding OpEx, we concentrate on energy consumption and component replacement, as well as costs for monitoring and fiber patching, which are specific to open access architectures. Other 


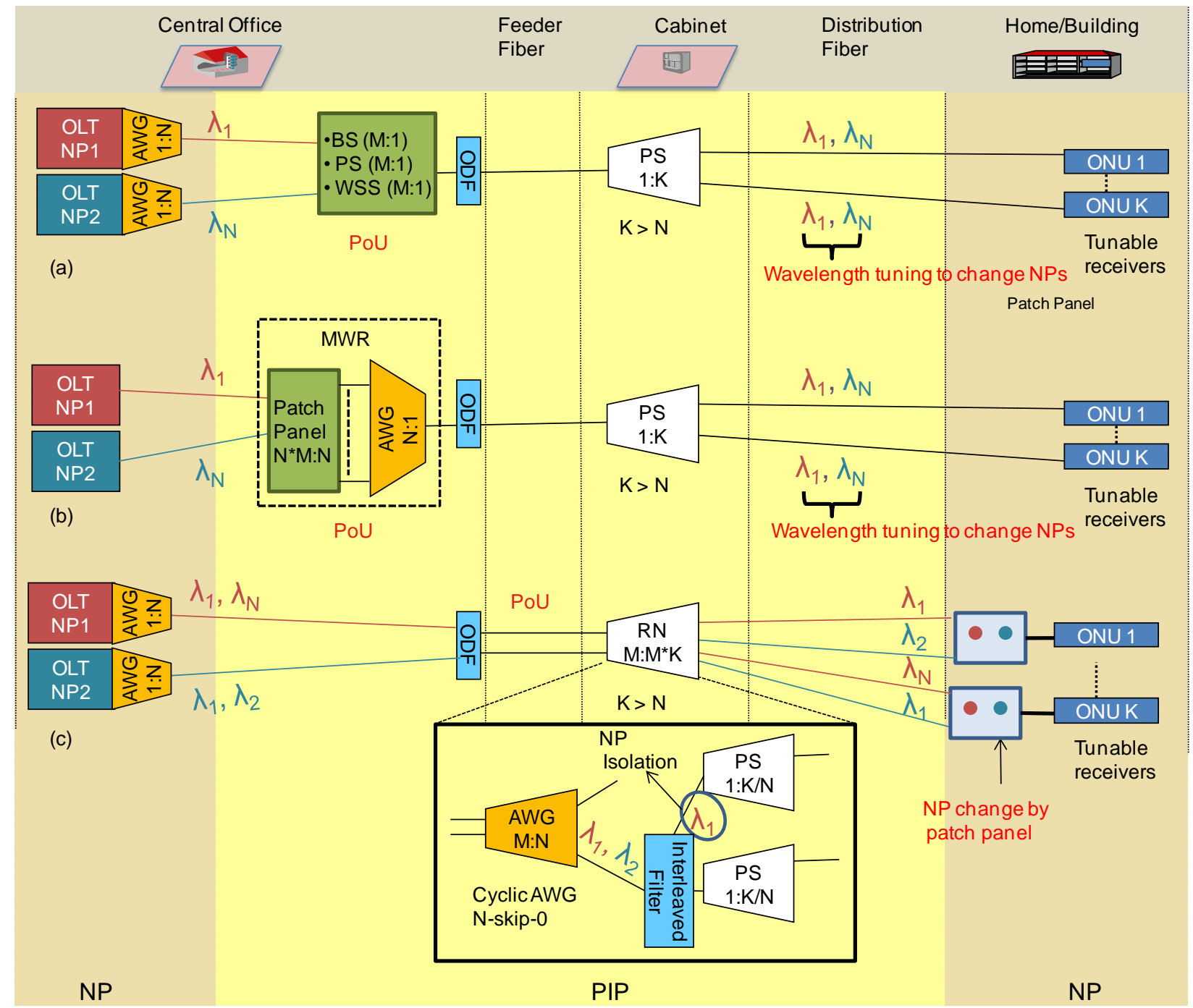

Figure 4: Wavelength Open access schemes at the OLT interface for TWDM-PON with point of unbundling (PoU) as: a) BS/PS/WSS b) MWR c) NP isolation

differences resulting from service provisioning are hard to quantify, and are not accounted. On the other hand, we do consider cost penalties due to insertion losses, which affect reach of the architectures, and consequently, node consolidation and the number of active sites.

The general parameters of the evaluation and the basic assumptions of the component cost, power consumption $(P C)$, insertion loss $(I L)$, and mean time between failures $(M T B F)$ are given in Table 1. For the OLT, we include shelf space, port card, transceiver (TRX), and layer 2 switching. For all cost calculations, we consider planning horizon or time span $\left(T_{s}\right)$ as 10 years, as this is a typical lifetime of active equipment technology [8]. These values have been discussed with the operators and the vendors in the European FP7 project OASE (optical access seamless evolution) [9]. A cost unit (CU) of 1 represents the cost of a GPON ONU. For the scheme with NP isolation, we assume the users to perform fiber patching.

\section{A. Cost parameters}

We evaluate the costs influenced by components and their replacement, power consumption, reach, monitoring and fiber patching. Apart from the component cost, we incorporate every design impact by translating it into its equivalent cost as follows:

- The cost of $P C$ of a component is evaluated as the product of $P C$ of a component, cost of power, and $T_{S}$.

- The cost of manual patching per user is 
evaluated as: $N_{h} \times T_{P} \times C_{M H} \times T_{S}$, where $N_{h}$ is the percentage of user churn (migration towards a different NP) in a year, $T_{P}$ is the time required for patching, and $C_{M H}$ is the cost of one manhour.

- The cost of monitoring can be calculated using the sum of the component cost (test equipment / number of users per PON) and the personal cost (number of full time equivalents $(\mathrm{FTE}) \times$ salary $\times T_{S} /$ number of users per $\mathrm{CO}$ ) spent on monitoring the spectrum compliance of NPs. Note that for calculating the personal cost, we used the number of users per $\mathrm{CO}$, as one person will not be dedicated for monitoring only a PON segment.

- The replacement cost of a component can be computed as the product of failure probability ( $T_{S} / \mathrm{MTBF}$ ) and the component cost.

- The reach of the technologies is decreased by the additional losses inserted by a PoU. The insertion loss of PoU and other components is given in Table 1 . The reach penalty affects the degree of node consolidation, and consequently cost, which is evaluated as in [10].

Further, we measure the variance of these costs with the adoption level and the customer churn rate.

Impact of adoption level - Only the subscribed customers will generate revenues to pay back the investment in the network. Subsequently, only those customers should be accounted when calculating the "effective cost per user."

The cost of deploying and maintaining the equipment, in most cases, cannot be purely linearly scaled with the number of users. Equipment located in the $\mathrm{CO}$ can be installed gradually according to the evolution of subscribed households, whereas for equipment located in the field (e.g. at the RN), there are not many possibilities of gradual installation (e.g., the PS located in the last mile should be installed as soon as there is one customer). An architecture that requires installation of equipment with a higher sharing granularity will therefore result in a relatively higher cost per user when the uptake of customers is lower than the optimal $100 \%$.

Impact of churn - A second economic influence that should be accounted is the impact of the churn rate. This churn rate is defined as the yearly percentage of users that switches to another NP. As this switching entails an extra cost, e.g. in the solution with MWR as PoU, the impact should be studied.

When a customer decides to change NP, he should be disconnected from the "old" NP and connected to the new one, which can be done manually in the case of an MWR, or automatically, through a simple reconfiguration of software for the other cases. It should be mentioned that the cost of churn is not limited to the manual or logical patching to disconnect and connect customers, but that it also entails some administrative costs (termination of contracts, final billing, setting up new contracts, etc.). Since these costs can be considered comparable in magnitude for all technology options under study, they were not taken into account in the current analysis. Currently, depending upon the region, the average churn rate in the telecommunication industry varies between $5 \%$ and $40 \%$ [11].

\section{B. Results}

Figure 5 shows the cost per user (expressed in CU) for the different architectures of wavelength open access in WDM-PON and TWDM-PON respectively, for an estimated uptake of $25 \%, 50 \%, 75 \%$, and $100 \%$ of the total household potential available in the area after 10 years, and with a churn rate of $25 \%$. Churn contributes to the cost in fiber re-patching for the solution with MWR as PoU. Other values of churn rates at which the solution corresponds to the cost of the cheapest solution are also indicated in the figure. Note that the cheapest solution can be different based on whether a scenario is fiber-rich or fiber-lean (e.g., in Figure 5: a, b and c) or can be the same in both the scenarios (e.g., in Figure 5 d).

The open access options at the $\mathbf{R N}$ interface are analyzed in Figure 5 (a) and (b). The solutions are more susceptible to adoption levels in the case of WDM-PON compared to TWDM-PON, with clearly higher adoption levels reduces the cost of the solutions.

In a fiber-lean scenario, the solution with DF as PoU is ruled out, and then the option with an ODF as PoU (Figure $2 \mathrm{~b}$ ) is the most economical choice for a churn rate lower than $42 \%$ in WDM- and TWDMPON (Figure 5 (a) and (b)). In this range of churn rate, the option with WAF is more costly due to the use of a WAF per customer (Figure $2 \mathrm{c}$ ). As a customer churn rate higher than $42 \%$ is mostly not expected, the options of wavelength open access at the RN interface will be limited.

In a fiber-rich deployment, the option with DF as PoU should be preferred for a churn rate high than $19 \%$ in WDM-PON and $37 \%$ in TWDM-PON. Hence, extra fibers must be deployed from the start for this range of churn rate.

The open access options at the OLT interface are analyzed in Figure 5 (c) and (d). As in the case of RN interface, the cost of the WDM-PON based 
architectures shows a higher susceptibility to adoption levels. For WDM-PON, the solution with MWR as PoU leads to the lowest cost, when the churn rate is lower than $33 \%$. We attribute this to the simplicity of the solution with no additional requirement of monitoring equipment, tunable receivers at the ONUs and complex PoUs. Cost of fiber patching seems to be not deterrent as the solution remains cost effective compared to other solutions even in a scenario when the churn rate is as high as $48 \%$ with an adoption level of $25 \%$. Thus, from a cost perspective this is an ideal candidate. The solution with multiple FF achieves the lowest cost for very high churn rates, e.g., a churn rate higher than $33 \%$ for an adoption level of $100 \%$; however, this solution can only be used in a fiber-rich scenario. The higher cost in this solution is due to the use of tunable receivers at the ONUs and multiple feeder fibers at the OLT. Whether this solution will still be used in a fiber-rich scenario depends upon the tradeoff between the costs of the solution vs. the potentially long migration times in the solution with MWR as PoU. Other solutions lead to significantly higher cost. The solution with PS as PoU has a high cost due to the requirement of monitoring. The solution with BS as PoU decreases the fan out, consequently sharing granularity, and uses tunable receivers at the ONUs. The solution with WSS as PoU leads to the highest cost due to the use of a WSS for a limited number of users in WDM-PON.

For TWDM-PON, the solution with a BS as PoU is most economical. The difference in the cost of the solution with normal TWDM-PON is within $1 \%$. This is attributed to the fact that TWDM-PON has a large fan out, and thus the cost of having an additional PoU is insignificant. The solution with WSS as PoU has the second best cost performance for churn rates higher than $20 \%$; it has an additional cost of between 3 and 10\% compared to the solution with BS as PoU. This can be regarded as a reasonable cost markup for added benefits offered by WSS as PoU, with respect to dynamic spectrum allocation among NPs. When compared with the solution with MWR as PoU, the solution achieves lower cost even when the churn rate is as low as $6 \%$ for adoption levels of $100 \%$. Moreover, given that its cost is inert to churn rates and given its capability to establish onthe-fly configurations, the solution with WSS as PoU will always be preferred. The solution with NP isolation can be used with an additional cost of between 13 to $28 \%$ compared to normal TWDMPON, given that the NP isolation is mandatory. This solution has a high cost due to its costly RN composed of multiple PSs and interleave filters.

The results in Figure 5 outline an increase in the cost for the NP layer in architectures supporting open access. While this in general is a "disadvantage" associated with sharing network, it does not weigh up to the savings that can be accrued because not all NPs are required to invest in the passive infrastructure anymore. What's more, even at the NP layer, though the cost per user increases, still the CapEx baggage per NP remains low, thanks to open access.

\section{CONCLUSIONS}

The combination of the ever-increasing demand for higher data rates with the trend towards open business models, asks that future-proof technologies be planned to cope with open access. This paper proposes architectures to enable open access in NGPON2 networks - WDM-PON and TWDM-PON using fiber and wavelength layers. We identified two interfaces at which the networks can be opened - RN and OLT. At the RN interface, the network can be opened using ODF, DF or WAF as PoU; at the OLT, the network can be opened using BS, MWR, WSS, PS, and FF as PoU. These solutions have their design tradeoffs. For example, the solutions using ODF and MWR require fiber patching and are sensitive to churn rate. The solutions with WAF and WSS use elements that are more complex, the solution with PS violates security, and the solution with BS cannot allocate the spectrum dynamically among NPs. On the other hand, the solutions with DF and FF require a fiber-rich scenario. Furthermore, in TWDM-PON, there are added security challenges, for broadcasting nature of PS violates inter-NP isolation, thus requiring a novel adaption in the RN of TWDM-PON (Figure 4 (c)).

Given the complexity of the design tradeoffs, there is no clear one-shoe-fit-all solution, and the selection requires an in-depth analysis of the impact of the design tradeoffs on the cost of the network. Following are the key findings of the cost analysis at these interfaces:

- RN Interface - The option with ODF as PoU leads to the lowest cost for churn rates lower than $19 \%$ in WDM-PON and $37 \%$ in TWDMPON. For higher churn rates, the solution with $\mathrm{DF}$ as $\mathrm{PoU}$ is preferred in a fiber-rich deployment.

- OLT Interface - For WDM-PON, the solution with MWR as PoU leads to the lowest cost. Whereas for TWDM-PON, the solution with BS as PoU is most cost effective. 


\section{ACKNOWLEDGEMENTS}

The research leading to these results has received funding from the European Community's Seventh Framework Programme (FP7/2007-2013) under grant agreement $n^{\circ} 249025$ (ICT-OASE). Marlies Van der Wee has received funding from the Agency for Innovation by Science and Technology (IWT) in Flanders.

\section{REFERENCES}

[1] V. Sivaraman, C. Russell, I. B. Collings, and A. Radford, "Architecting a national optical fiber open-access network: The Australian Challenge," IEEE Network vol. 26, no. 4, pp. 4-10, 2012.

[2] Y. Luo et al., "Time and Wavelength Division Multiplexed Passive Optical Network (TWDM-PON) for Next Generation PON Stage 2 (NG-PON2)," IEEE Journal of Lightwave Technology, vol. 31, no. 4, pp. 587 - 593, 2013.

[3] K. Grobe, M. Roppelt, A. Autenrieth, J. P. Elbers, and M Eiselt, "Cost and Energy Consumption Analysis of Advanced WDM-PONs," IEEE Communications Magazine, vol. 49, no. 2, pp. s25 - s32, 2011.

[4] M. Van der Wee et al., "Making a success of FTTH learning from case studies in Europe," Journal of the Institute of Telecommunications Professionals, vol. 5, no. 4, pp. 22-31, 2011.

[5] OASE Project, D6.3: Value network evaluation, Jan. 3013, available at: http://www.ict-oase.eu/index.php?page $=120 \&$

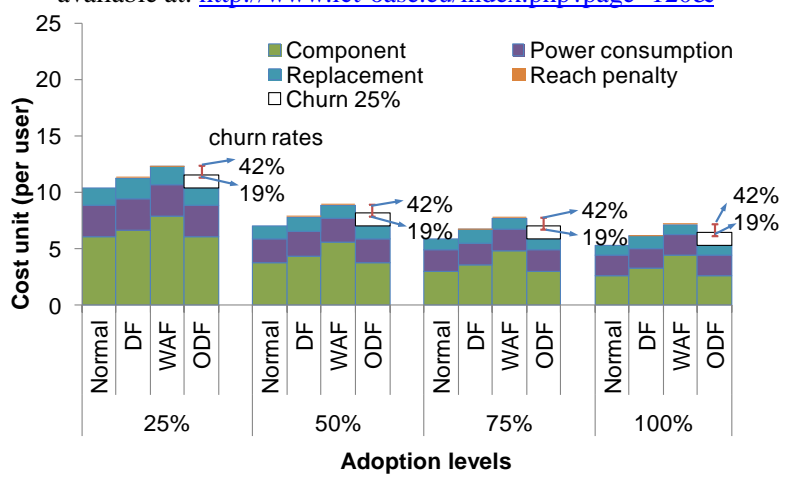

a) WDM-PON, RN interface

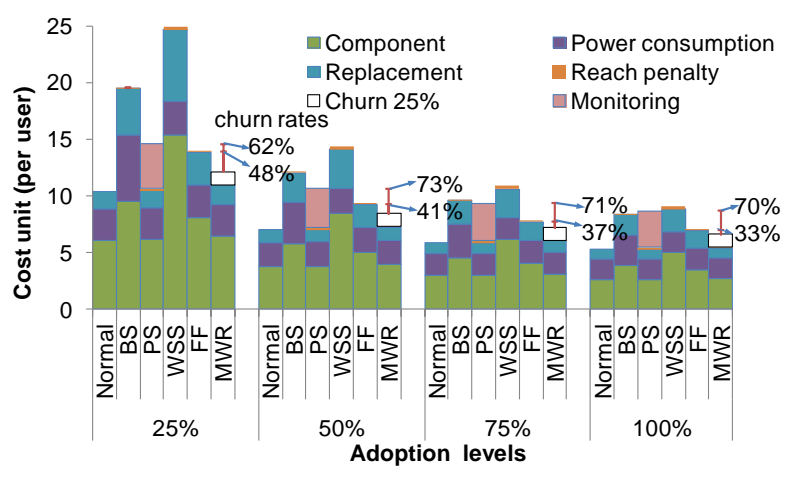

c) WDM-PON, OLT interface
[6] J. S. Wey, H. Rohde, and C. Badstieber. "Open lambda initiative for ultra high capacity optical access networks," Access Networks and In-house Communications. Optical Society of America, 2010.

[7] K. Casier, S. Verbrugge, R. Meersman, D. Colle, M. Pickavet, P. Demeester, "A clear and balanced view on FTTH deployment costs," Journal of the Institute of Telecommunications Professionals, vol. 2, no. 3, pp.27-30, 2008.

[8] M. Van der Wee et al., "Techno-Economic Evaluation of FTTH Migration for a Network Provider: Comparison of NG-AON and TWDM-PON," in Proc. of ACP, 2013.

[9] OASE Project, D4.2.1: Technical assessment and comparison of next-generation optical access system concepts, Oct. 2011, available at: $h$ ttp://www.ict-oase.eu/index.php?page $=120 \&$

[10] C. Lange, R. Hülsermann, D. Kosiankowski, F. Geilhardt, and A. Gladisch, "Effects of network node consolidation in optical access and aggregation networks on costs and power consumption," In OPTO, pp. 76210F-76210F, International Society for Optics and Photonics, 2010.

[11] T. Bobbier, "Keeping the Customer Satisfied: The Dynamics of Customer Defection, and the Changing Role of the Loss Adjuster," CILA Report, http://www.cila.co.uk/publication/articles/keeping-customersatisfied-tony-boobier, accessed July 2013.

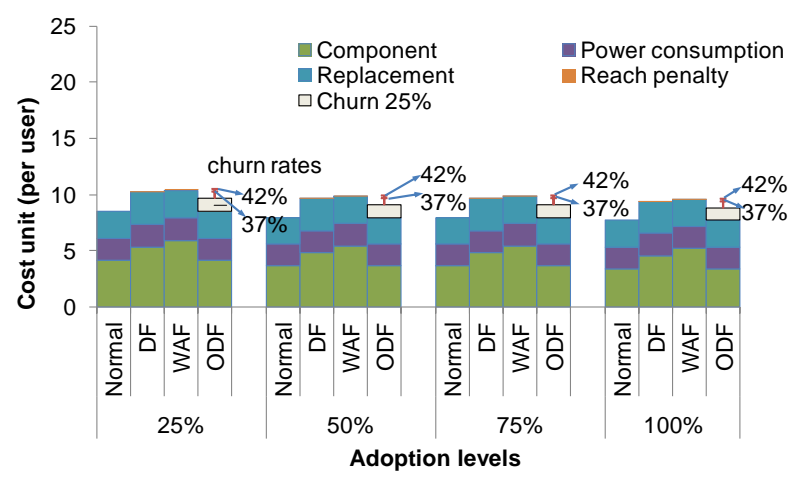

b) TWDM-PON, RN interface

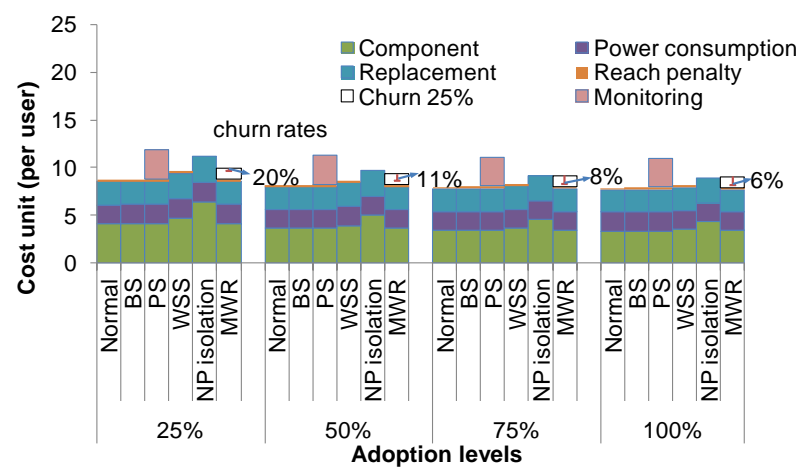

d) TWDM-PON, OLT interface

Figure 5: Cost evaluation of different architectures for wavelength open access in WDM-PON and TWDM-PON with varying adoption levels (varied from $25 \%$ to $100 \%$ ) and churn rates (varied to correspond to the costs of the cheapest options, further split between fiberlean and fiber-rich scenarios for the architectures in $a, b, c$ and $d)$. 
Table 1: General parameters and assumptions of cost evaluation.

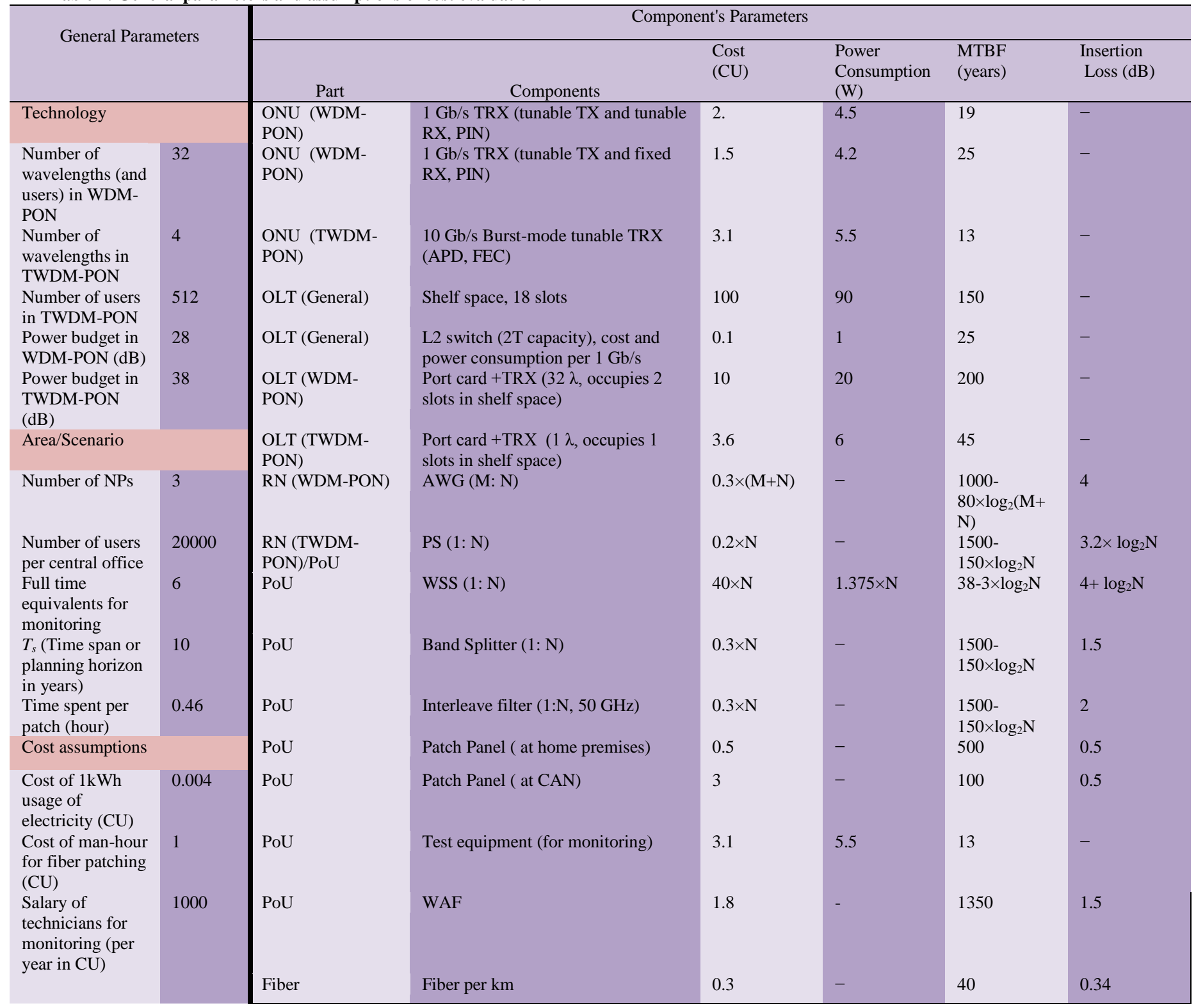

Abbreviations used in the table: FEC: forward error correction; TEC: thermal electric control; APD: Avalanche Photodiode; TRX: transceiver; T: terabit. The numbers used in the table are from [4] and [6]. 